\title{
Outcomes of Robot-assisted Laparoscopic Gynecological Surgery
}

\author{
Munazzah Rafique, Tehmina Aziz and Sahar Al-Suwailem \\ Women Specialized Hospital, King Fahad Medical City, Riyadh, Saudi Arabia
}

\begin{abstract}
Objective: To compare the outcomes of robot-assisted (RA) and standard laparoscopic gynecological surgery (S-LGS) in a tertiary care hospital, and evaluate the factors affecting the outcomes of RA-LGS to identify areas of improvement.

Study Design: A descriptive study.

Place and Duration of Study: King Fahad Medical City, Riyadh, Saudi Arabia, from 2013 to 2018.

Methodology: In this 5-year retrospective study, 65 LGS cases, including 37 RA-LGS and 28 S-LGS, in a single tertiary care hospital, were included. Demographic data, clinical pathological details, and complications of the cases were recorded. Surgeons performing RA-LGS were also interviewed regarding their training/experience, competency of surgical assistance, and suggestions for improving training.

Results: Operative times ( $3.70 \pm 0.96$ vs. $2.07 \pm 0.78 \mathrm{~h}, \mathrm{p}<0.001)$ and hospital stays (3.53 \pm 3.29 vs. $1.96 \pm 1.34$ days, $p=0.022$ ) were significantly longer in the RA-LGS group than in the S-LGS group. Intraoperative complications, which were primarily adjacent organ damage $(21.6 \%$ vs. $0.0 \%, p=0.029)$, were significantly more common in the RA-LGS group. There were no significant differences between the groups in terms of the need to convert to laparotomy, immediate/late postoperative complications, estimated blood loss, or the need for blood transfusion. The interview survey results suggested the lack of a trained team assisting in RA-LGS, as the reason for the poor outcomes.

Conclusion: There were no advantages of RA-LGS over S-LGS. Longer training periods for RA-LGS, with minimum 20-50 cases as part of a structured training programme, may improve outcomes.
\end{abstract}

Key Words: Laparoscopic surgeries, Robot-assisted surgical procedures, Gynecological disease, Uterine cancer, Menorrhagia.

How to cite this article: Rafique M, Aziz T, Al-Suwailem S. Outcomes of robot-assisted laparoscopic gynecological surgery. J Coll Physicians Surg Pak 2020; 30(3):254-258.

\section{INTRODUCTION}

Compared with laparotomy, the use of laparoscopic technology for gynecological procedures has resulted in significant patient benefits, including decreased blood loss, shorter hospital stays, faster recovery, improved cosmesis and decreased postoperative pain.1,2 Approximately 500,000 women undergo elective laparoscopic hysterectomy in the United States alone, with the majority $(87 \%)$ being for benign uterine pathologies. ${ }^{2}$ Laparoscopic gynecological procedures are indicated for several conditions. For example, intractable abnormal uterine bleeding (AUB) is an indication for laparoscopic hysterectomy. ${ }^{3}$ Similarly, laparoscopic procedures are used for treating cases of uterine fibroids after failure of conservative therapy. ${ }^{4}$ Laparoscopy has also revolu-tionised the treatment of ovarian cysts, another common indication for gynecological surgery. 3,4

The advantages of the laparoscopic approach include minimising tissue trauma and maintaining moistness in

Correspondence to: Dr. Munazzah Rafique, Women Specialized Hospital, King Fahad Medical City, PO Box 59046, Riyadh

11525, Saudi Arabia

E-mail:munazzahr@yahoo.com

Received: June 25, 2019; Revised: January 30, 2020;

Accepted: January 30, 2020 the tissues, thereby decreasing the possibility of adhesion formation. 5 Nevertheless, laparoscopic techniques have inherent drawbacks that result in their slow incorporation into the surgical practices of most gynecologists. ${ }^{6}$ Limitations imposed by two-dimensional vision, instrument rigidity, and counterintuitive movements were among the reasons that have been proposed to slow the widespread adoption of laparoscopic techniques. ${ }^{7}$ The steep learning curve for surgeons and longer operative times along with ergonomic difficulty and tremor amplification are major obstacles in the widespread acceptance and application of minimally invasive surgical techniques in gynecological surgery.6,7

Numerous studies comparing standard laparoscopic gynecological surgery (S-LGS) and robot-assisted lyparoscopic gynecological surgery (RA-LGS) have shown improved surgical accuracy, faster intracorporeal knot tying, reduced skill-based errors and shorter time required for learning. ${ }^{5-8}$ However, there is a lack of data regarding RA surgeries from developing nations, where training is necessary to improve the frequency of use and outcomes of such technologies.

Therefore, the primary aim of the present study was to compare the outcomes, particularly short-term ones, and the complications between these procedures in a tertiary care facility in Saudi Arabia. 


\section{METHODOLOGY}

A retrospective descriptive study was done after obtaining Institutional Review Board's approval of IRB Registration with OHRP/NIH, USA IRB00010471 (Log number 18-440). The study was performed in accordance with the ethical standards and the need for informed consent was waived by the Institutional Review Board. Moreover, after data collection, patient identifiers were removed before data analysis to keep the confidentiality and privacy of patient information. From the hospital records, patients undergoing RA-LGS at the Women's Specialized Hospital at King Fahad Medical City, Riyadh, from 2013 to 2018, were selected. Patients undergoing S-LGS in the same time period were selected for comparison. The inclusion criteria were as follows: elective surgeries for abdominal mass, adnexal mass, uterine fibroids, menorrhagia, perimenopausal bleeding, and stage 1 endometrial cancer. The exclusion criteria were: patients undergoing emergency procedures and patients with stage 2 and above endometrial cancer, infertility, intra-uterine contraceptive device (IUCD) insertion, and urogynecological procedures such as sacrocolpopexy and colposuspension. All patients had undergone laparoscopic or da Vinci laparoscopic hysterectomy, myomectomy, cystectomy, or oophorectomy and lymph node dissection. Patients with incomplete records were also excluded. The choice between RA-LGS and S-LGS was made on an individual case basis by the surgeons.

Perioperative antibiotics were administered to all patients in both groups. Lower extremity compression devices and elastic stockings were used for deep venous thrombosis prophylaxis. All procedures were performed under general anesthesia. Thereafter, S-LGS following standard procedures were performed by surgeons with experience in laparoscopic surgery. On the other hand, RA-LGS was performed by surgeons with experience in laparoscopic surgery who received training to operate the da Vinci surgical system through the master control in the console, and an assistant and a scrub nurse stood at the patient's side.

Operative variables such as operative time, complications, conversion to laparotomy, and blood loss, were recorded. In addition, postoperative pain assessment, need for analgesics, days to recovery, and discharge time, were noted. Patients were followed up for six weeks postoperatively to evaluate their recovery and postoperative outcomes. This duration was chosen because it is the standard postoperative follow-up period recommended for any late procedure-related complications. Patients with a blood loss of $>200 \mathrm{~mL}$ were offered postoperative hemoglobin test at the 4-6-week visit. The reviewing physician qualitatively evaluated patient satisfaction levels by asking questions about recovery, new complaints, bowel and bladder functions, sexual functions, and overall satisfaction. Qualitative surgeon satisfaction levels were also recorded. Validated interview question survey was used for gathering detailed information from surgeons performing RA-LGS regarding related issues. Specifically, one-on-one, faceto-face interviews, including open-ended questions, were conducted for the surgeons' experiences, technical hitches faced, and gaps identified in RA surgeries.

Categorical variables are presented as numbers and percentages, and continuous variables are expressed as mean \pm standard deviation (SD). Independent sample t-tests were applied to test the mean significant differences between the two groups in terms of patient characteristics. Pearson's Chi-square or Fisher's exact tests were applied depending on whether the cell expected frequency was $<5$, and these were used to determine the significant relationship between categorical variables. A p-values of $<0.05$ were considered statistically significant. All data were entered and analysed through the statistical package SPSS 22 (SPSS Inc., Chicago, IL, USA).

\section{RESULTS}

Overall, 65 patients with an average age of $45.2 \pm 11.9$ (range, 15-66) years were enrolled (Table I). Of these, $37(56.9 \%)$ underwent RA-LGS and $28(43.1 \%)$ underwent S-LGS. The average patient age was significantly higher in the RA-LGS group than in the S-LGS group (49.16 \pm 8.54 vs. $39.96 \pm 13.09$ years, $p=0.002$ ). The average operative time was $3 \pm 1.2$ (range 0.41-6.54) hours and average length of hospital stay was 2.84 \pm 2.73 (range, 1-17) days (Table I).

Table I: Comparative analysis of robot-assisted and standard laparoscopic surgeries with respect to continuous variables $(n=65)$.

\begin{tabular}{l|c|c|c}
\hline Characteristics & $\begin{array}{c}\text { Robot-assisted } \\
(\text { Mean } \pm \text { SD })\end{array}$ & $\begin{array}{c}\text { Standard laparoscopy } \\
(\mathrm{n}=37)\end{array}$ & $\begin{array}{c}\mathrm{p} \text {-value } \\
(\mathrm{n}=28)\end{array}$ \\
\hline Age & $49.16 \pm 8.54$ & $39.96 \pm 13.09$ & ${ }^{*} 0.002$ \\
BMI & $32.82 \pm 5.24$ & $31.62 \pm 7.70$ & 0.484 \\
Operative time & $3.70 \pm 0.96$ & $2.07 \pm 0.78$ & ${ }^{*}<0.001$ \\
Length of stay in hospital & $3.53 \pm 3.29$ & $1.96 \pm 1.34$ & ${ }^{*} 0.022$ \\
\hline
\end{tabular}

The comorbid medical and surgical conditions in both groups were hypertension and diabetes mellitus. Moreover, the most frequent surgical procedure in both groups was cesarean section. The most common indications for surgery were AUB (54.1\%); and in the RA-LGS group, it was abdominal mass and pain $(57.1 \%)$ (Table II). The reason for conversion to laparotomy was adjacent organ damage to either the intestine or bladder for RA-LGS and inability to perform the target procedure and bleeding for S-LGS.

The operative time was significantly longer in the RA-LGS group than in the S-LGS group (3.70 \pm 0.96 vs. 2.07 \pm 0.78 hours, $p<0.001)$. The hospital stay was also significantly longer in the RA-LGS group than in the S-LGS group ( $3.53 \pm 3.29$ vs. $1.96 \pm 1.34, p=0.022)$ (Table II). 
Patient and surgeon satisfaction levels were similar between both the groups at the 4-6-week postoperative follow-up visit. Follow-up was discontinued in most patients because recovery was uneventful. However, oncology patients were asked to follow up as per the

Table II: Comparative analysis of robot-assisted and standard laparoscopic surgeries with respect to categorical variables $(n=65)$.

\begin{tabular}{|c|c|c|c|}
\hline Characteristics & $\begin{array}{l}\text { Robot- } \\
\text { assisted }\end{array}$ & $\begin{array}{c}\text { Standard } \\
\text { laparoscopy }(\mathrm{n}=28)\end{array}$ & $p$-value \\
\hline \multicolumn{4}{|l|}{ History } \\
\hline AUB & $20(54.1 \%)$ & $6(21.4 \%)$ & ${ }^{*}<0.001$ \\
\hline Endometrial cancer & $6(16.2 \%)$ & $0(0.0 \%)$ & \\
\hline Fibroid uterus & $6(16.2 \%)$ & $0(0.0 \%)$ & \\
\hline Ovarian cyst & $2(5.4 \%)$ & $0(0.0 \%)$ & \\
\hline Postmenopausal bleeding & $3(8.1 \%)$ & $6(21.4 \%)$ & \\
\hline Abdominal mass and pain & $0(0.0 \%)$ & $16(57.1 \%)$ & \\
\hline \multicolumn{4}{|l|}{ Past medical history } \\
\hline Yes & $20(54.1 \%)$ & $9(32.1 \%)$ & 0.078 \\
\hline No & $17(45.9 \%)$ & $19(67.9 \%)$ & \\
\hline \multicolumn{4}{|l|}{ Previous surgery } \\
\hline Yes & $23(62.2 \%)$ & $14(50.0 \%)$ & 0.327 \\
\hline No & $14(37.8 \%)$ & $14(50.0 \%)$ & \\
\hline \multicolumn{4}{|l|}{ Planned procedure } \\
\hline Hysterectomy + cystoscopy & $31(83.8 \%)$ & $8(28.6 \%)$ & $<0.001$ \\
\hline Myomectomy & $6(16.2 \%)$ & $3(10.7 \%)$ & \\
\hline Oophorectomy/cystectomy & $0(0.0 \%)$ & $17(60.7 \%)$ & \\
\hline \multicolumn{4}{|l|}{ Indication for surgery } \\
\hline Adenomyosis & $2(5.4 \%)$ & $1(3.6 \%)$ & $<0.001$ \\
\hline Abnormal uterine bleeding & $12(32.4 \%)$ & $0(0.0 \%)$ & \\
\hline Endometrial cancer & $6(16.2 \%)$ & $6(21.4 \%)$ & \\
\hline Fibroid uterus & $12(32.4 \%)$ & $3(10.7 \%)$ & \\
\hline Ovarian cyst & $2(5.4 \%)$ & $17(60.7 \%)$ & \\
\hline Postmenopausal bleeding & $3(8.1 \%)$ & $0(0.0 \%)$ & \\
\hline Uterine polyp & $0(0.0 \%)$ & $1(3.6 \%)$ & \\
\hline \multicolumn{4}{|l|}{ Morcellation } \\
\hline Done & $11(29.7 \%)$ & $1(3.6 \%)$ & ${ }^{*} 0.007$ \\
\hline Not done & $26(70.3 \%)$ & $27(96.4 \%)$ & \\
\hline \multicolumn{4}{|l|}{ Conversion to laparotomy } \\
\hline Laparotomy & $2(5.4 \%)$ & $2(7.1 \%)$ & 0.773 \\
\hline NA & $35(94.6 \%)$ & $26(92.9 \%)$ & \\
\hline \multicolumn{4}{|l|}{ Blood transfusion } \\
\hline 2 units of PRBC & $3(8.1 \%)$ & $1(3.6 \%)$ & 0.451 \\
\hline None & $34(91.9 \%)$ & $27(96.4 \%)$ & \\
\hline \multicolumn{4}{|l|}{ Operative complications } \\
\hline Bleeding & $3(8.1 \%)$ & $2(7.1 \%)$ & ${ }^{*} 0.029$ \\
\hline Damage to adjacent organs & $8(21.6 \%)$ & $0(0.0 \%)$ & \\
\hline None & $26(70.3 \%)$ & $26(92.9 \%)$ & \\
\hline \multicolumn{4}{|l|}{$\begin{array}{l}\text { Immediate postoperative } \\
(0-3 \text { weeks) complications }\end{array}$} \\
\hline Anemia & $1(2.7 \%)$ & $3(10.7 \%)$ & 0.397 \\
\hline UTI & $2(5.4 \%)$ & $1(3.6 \%)$ & \\
\hline None & $34(91.9 \%)$ & $24(85.7 \%)$ & \\
\hline \multicolumn{4}{|l|}{$\begin{array}{l}\text { Late postoperative (3-6 weeks) } \\
\text { complications }\end{array}$} \\
\hline Pelvic collection & $1(2.7 \%)$ & $0(0.0 \%)$ & 0.218 \\
\hline Urinary problem & $3(8.1 \%)$ & $0(0.0 \%)$ & \\
\hline Intra-abdominal abscess & $0(0.0 \%)$ & $1(3.6 \%)$ & \\
\hline None & $33(89.2 \%)$ & $27(96.4 \%)$ & \\
\hline
\end{tabular}

$U T I=$ Urinary tract infection, $P R B C=$ Packed red blood cells, BMI $=$ Body mass index $E B L=$ Estimated blood loss, $A U B=$ Abnormal uterine bleeding, SD: Standard deviation * Indicates that $p<0.05$ is statistically significant. protocol for the monitoring of cancer recurrence and long-term surveillance (Table II).

Surgeons involved in RA-LGS considered RA surgeries to have an important role in gynecologic surgery and in helping surgeons to offer state-of-the-art, evidencebased technology. According to them, RA surgery has revolutionised the practice of gynecology. The most important factors affecting the outcome of RA surgery was the lack of a trained team assisting during the surgery and the longer learning period required for robotic surgery. Most surgeons had performed an average of 50 cases as part of their training before becoming procedurally independent. Appropriate structured training programmes were recommended for the whole team involved in the RA surgeries.

\section{DISCUSSION}

In the present single-centre retrospective study, which aims to address the lack of information regarding RA surgery outcomes from developing nations, the outcomes of S-LGS and RA-LGS in a tertiary care hospital in Saudi Arabia were compared. The results showed that RA-LGS required significantly longer operative time and hospital stay than S-LGS. In addition, intraoperative complications were significantly more common in RA-LGS than in S-LGS. These complications were mostly the reasons for the damage to adjacent organs. Several studies have compared various aspects of RA-LGS and S-LGS for various gynecological entities. ${ }^{5-8}$ For example, Ind et al. 9 performed a systematic review and meta-analysis comparing RA-LGS and S-LGS for the treatment of endometrial cancer. They found no differences in terms of operative time; however, hospital stays were significantly shorter in the RA-LGS group. In addition, estimated blood loss, conversions to laparotomy, and complications were reduced in the RA-LGS group. However, the RA-LGS approach was significantly more expensive. Albright et al. ${ }^{1}$ performed a systematic review and meta-analysis of randomised trials of RA-LGS vs. S-LGS hysterectomies for benign disease. No significant differences were observed with respect to complications between the two approaches. Although they could not analyse secondary outcomes because of heterogeneity, they nevertheless found no significant differences in hospital stay, total operative time, conversion to laparotomy, or estimated blood loss.

Lonnerfors compared RA-LGS with S-LGS for the treatment of uterine fibroids and found similar results in terms of short-term outcomes for RA myomectomies than for laparoscopic procedures. ${ }^{10}$ Postoperative fertility rates were similar following myomectomy for both RA-LGS and S-LGS. Chen et al. performed a metaanalysis comparing RA-LGS and S-LGS only for the staging of endometrial cancer. ${ }^{11}$ RA-LGS was less frequently converted to laparotomy, produced lower complication rates, had lower estimated blood loss, and 
had shorter hospital stays. Magrina found that the RA group had a significantly shorter operative time, less estimated blood loss, and shorter hospital stay. ${ }^{12}$

RA technology was designed to facilitate laparoscopy and to improve surgeons' laparoscopic skills by eliminating the technological drawbacks of laparoscopy, particularly by providing three-dimensional vision, instrument articulation, and intuitive movements. ${ }^{13}$ Other important additions were surgeon comfort (by providing the surgeon a console, which can be used to perform the operation while sitting down and which can decrease tremor and downscale movements, all of which improve surgeons' precision). ${ }^{14}$ The da Vinci surgical system was designed for complex procedures in small spaces. ${ }^{15}$ Therefore, manipulating large specimens and requiring a wide operating field are not optimal uses for the device. ${ }^{11}$ RA surgery can transform laparoscopic surgery by providing, for the first time, instruments with distal ends that mimic the intricate movements of the human hand while providing the surgeon with a high-definition, three-dimensional view of the operative field. ${ }^{16}$ With the growth and advancement of this technology, further refinement and improvements are expected, which could allow for even more precise and even less invasive surgical options beyond laparoscopy.17 The introduction of robotics has resulted in a rapid decrease in the frequency of open hysterectomy, which was not observed after the launch of laparoscopy this demonstrates surgeon preference for robotics over laparoscopic technology. ${ }^{17,18}$ The high purchase cost, even higher purchase cost of fresh instruments (with only 10 uses), and annual maintenance fee prevent its widespread use. ${ }^{17}$

Unlike the results of previous studies, this study showed poorer outcomes for RA-LGS than for S-LGS. This finding, however, is supported by the results of the qualitative analysis of the interview survey, in which surgeons agreed with the importance of RA surgery in the advancement of gynecological surgery, but revealed gaps such as the need for structured training programmes for the whole team involved and for longer learning periods with higher number of cases for procedural independence. These findings are important in the context of developing nations, considering the insufficient budgets and infrastructure for healthcare delivery. ${ }^{19}$ RA surgery is yet to be established in such geographies, and the lack of availability of RA surgical systems and consequent gaps in specialised training may affect the outcome of RA surgeries. ${ }^{20}$

This study had several limitations. First, because of its retrospective nature, selection bias may have affected the result. Second, several demographic variables were not matched between the RA-LGS and S-LGS groups. These include the type of surgeries performed and patient age. This may have resulted in the unfavourable outcomes for RA-LGS. Third, as demonstrated in the interview survey, inclusion of cases in which the surgery was performed after a longer training period or by a trained assisting surgical team could improve outcomes. Nevertheless, the study findings report the relatively poor outcomes of RA-LGS, especially in developing nations. Future, well-designed, prospective studies with well-defined long-term clinical outcomes, including complications, cost, pain, return to normal activity, and quality of life, are necessary to extend our findings and validate the usefulness of this new technology.

\section{CONCLUSION}

Authors' experience with laparoscopic treatment of gynecological conditions suggests that RA assistance provides no advantage over S-LGS. Complication rates were significantly greater in the RA-LGS group than in the S-LGS group. The qualitative analysis of the interview survey revealed the lack of structured training of the team performing RA-LGS and the need for longer training periods for improving outcomes of RA-LGS. These gaps need to be addressed for improving the outcomes of RA-LGS in developing nations.

\section{ETHICAL APPROVAL:}

Study was done after obtaining Institutional Review Board approval of IRB Registration with OHRP/NIH, USA IRB00010471 (Log number 18-440).

\section{PATIENTS' CONSENT:}

Not applicable as retrospective study. Moreover, patient identifiers were removed before data analysis for confidentiality.

\section{CONFLICT OF INTEREST:}

Authors declared no conflict of interest.

\section{AUTHORS' CONTRIBUTION:}

MR: Conceptualised the project and draft development, data collection, basic manuscript writing.

TA: Did drafting and revised it critically for important intellectual content.

SS: Helped in data revalidation, manuscript proofreading, editing, final approval of the version to be published.

\section{REFERENCES}

1. Albright BB, Witte T, Tofte AN, Chou J, Black JD, Desai VB, et al. Robotic versus laparoscopic hysterectomy for benign disease: A systematic review and meta-analysis of randomized trials. J Minim Invasive Gynecol 2015; 23:18-27.

2. Jin YM, Liu SS, Chen J, Chen YN, Ren CC. Robotic radical hysterectomy is superior to laparoscopic radical hysterectomy and open radical hysterectomy in the treatment of cervical cancer. PLoS One 2018; 13:e0193033.

3. Munro MG, Dickersin K, Clark MA, Langenberg P, Scherer RW, Frick KD. The surgical treatments outcomes project for dysfunctional uterine bleeding: summary of an agency for 
health research and quality-sponsored randomized trial of endometrial ablation versus hysterectomy for women with heavy menstrual bleeding. Menopause 2011; 18:451-8.

4. Mettler L, Schollmeyer T, Tinelli A, Malvasi A, Alkatout I. Complications of uterine fibroids and their management, surgical management of fibroids, laparoscopy and hysteroscopy versus hysterectomy, haemorrhage, adhesions, and complications. Obstet Gynecol Int 2012; 2012:791248.

5. Quaas AM, Einarsson JI, Srouji S, Gargiulo. Robotic myomectomy: A review of indications and techniques. Rev Obstet Gynecol 2010; 3:185-91.

6. Soliman PT, Iglesias D, Munsell MF, Frumovitz M, Westin SN, Nick AM, et al. Successful incorporation of robotic surgery into gynecologic oncology fellowship training. Gynecol Oncol 2013; 131:730-3

7. Sarle R, Tewari A, Shrivastava A. Surgical robotics and laparoscopic training drills. J Endourol 2004; 18:63-6.

8. Sinha R, Sanjay M, Rupa B, Kumari S. Robotic surgery in gynecology. J Minim Access Surg 2015; 11:50.

9. Ind T, Laios A, Hacking M, Nobbenhuis M. A comparison of operative outcomes between standard and robotic laparoscopic surgery for endometrial cancer: A systematic review and meta-analysis. Int J Med Robot 2017; 13:e1851.

10. Lonnefors C. Robot-assisted myomectomy. Best Pract Res Clin Obstet Gynaecol 2018; 46:113-9.

11. Chen SH, Li ZA, Huang R, Xue HQ. Robot-assisted versus conventional laparoscopic surgery for endometrial cancer staging: A meta-analysis. Taiwan J Obstet Gynecol 2016; 55: 488-94.

12. Magrina JF, Zanagnolo VL. Robotic surgery for cervical cancer. Yonsei Med J 2008; 49:879-85.

13. Bouquet de Joliniere J, Librino A, Dubuisson JB, Khomsi F, Ben Ali N, Fadhlaoui A, et al. Robotic surgery in gynecology. Front Surg 2016; 3:26.

14. Tinelli A Laparoscopic entry: Traditional methods, new insights and novel approaches. Springer Science \& Business Media; 2011.

15. Dakin GF, Gagner M. Comparison of laparoscopic skills performance between standard instruments and two surgical robotic systems. Surg Endosc 2003; 17:574-9.

16. Prasad SM, Prasad SM, Maniar HS, Chu C, Schuessler RB, Damiano RJ Jr. Surgical robotics: Impact of motion scaling on task performance. J Am Coll Surg 2004; 199:863-8.

17. Lanfranco AR, Castellanos AE, Desai JP, Meyers WC. Robotic surgery: A current perspective. Ann Surg 2004; 239:14-21.

18. Kho RM, Hilger WS, Hentz JG, Magtibay PM, Magrina JF. Robotic hysterectomy: Technique and initial outcomes. Am J Obstet Gynecol 2007; 197:113.e1-113.

19. Chang L, Satava RM, Pellegrini CA, Sinanan MN. Robotic surgery: Identifying the learning curve through objective measurement of skill. Surg Endosc 2003; 17:1744-8.

20. Yohannes $P$, Rotariu $P$, Pinto $P$, Smith $A D$, Lee BR. Comparison of robotic versus laparoscopic skills: Is there a difference in the learning curve? Urology 2002; 60:39-45. 